Diskussionspapier Nr. 98-14

\title{
Innovationsstrategien und Forschungsaktivitäten ostdeutscher Unternehmen
}

Johannes Felder und Alfred Spielkamp 


\section{Das Wichtigste in Kürze}

Dieser Beitrag untersucht die Innovationsstrategien und Forschungs- und Entwicklungsaktivitäten (FuE) ostdeutscher Unternehmen sowie mögliche Innovationsdefizite in diesem Zusammenhang. Als Hinweis auf die zu geringen Innovationsaktivitäten ostdeutscher Unternehmen werden häufig die niedrigen FuEAufwendungen der Wirtschaft in den neuen Bundesländern angesehen. Doch die geringe FuE-Intensität auf aggregierter Ebene spiegelt lediglich die nachteilige ostdeutsche Wirtschafts- und Unternehmensgrößenstruktur wider. Eine Untersuchung des FuE-Verhaltens von Unternehmen auf der Basis des Mannheimer Innovationspanels zeigt, daß bei Berücksichtigung der ostdeutschen Wirtschaftsund Unternehmensgrößenstrukturen die Unternehmen im Verarbeitenden Gewerbe Ostdeutschlands häufiger und mit einer höheren Intensität FuE durchführen als (vergleichbare) westdeutsche Unternehmen. Auch bei anderen Innovationsindikatoren, wie z.B. dem Anteil der Unternehmen mit Basis- oder Verbesserungsinnovationen oder der Höhe der über FuE hinausgehenden Innovationsaufwendungen, läßt sich auf der Unternehmensebene kein Rückstand feststellen.

Problematisch ist dabei, daß viele ostdeutsche Unternehmen schon zu Beginn des wirtschaftlichen und technologischen Aufholprozesses FuE mit hoher Intensität durchführen, und die Forschungsaktivitäten dem ökonomischen Erfolg, gemessen an der Arbeitsproduktivität der Unternehmen, vorauseilen. Erfolgversprechender scheint dagegen der Weg von ostdeutschen Unternehmen zu sein, die zunächst ohne eigene FuE über Technologietransfers und hohe Investitionen versucht haben, den technologischen und wirtschaftlichen Rückstand zu reduzieren. Dieser Weg wurde insbesondere von Tochterunternehmen westdeutscher oder ausländischer Firmen gewählt.

Eine wichtige, zukünftige technologiepolitische Herausforderung dürfte es daher sein, den Unternehmen aus der ersten Gruppe beim Aufbau der wirtschaftlichen Voraussetzungen für erfolgreiche FuE zu helfen. Wichtig ist es, daß es diesen Unternehmen gelingt, eine effiziente Produktion aufzubauen. Daneben muß Kundenorientierung zur prioritären Aufgabe werden. Die Integration in das gesamtdeutsche Innovationssystem ist schließlich für die Unternehmen von hoher Bedeutung, damit zum einen der Zugang zu unternehmensexternem Wissen verbessert wird und zum anderen bestehende Vertriebswege genutzt werden können. 


\title{
Innovationsstrategien und Forschungsaktivitäten ostdeutscher Unternehmen
}

\author{
von \\ Johannes Felder und Alfred Spielkamp \\ Zentrum für Europäische Wirtschaftsforschung (ZEW)
}

April 1998

\begin{abstract}
This paper deals with the innovation behavior and the R\&D activities at the firm level in East Germany. The theoretical and empirical analyses lead to the conclusion that the relatively low level of the R\&D intensity in East Germany is a reflex of the relatively weak economy, especially the disadvantages due to the industry structure and the average firm size. However, taken these 'disadvantages' on the aggregate level into account a great number of east german companies engage more often in and devote more ressources to $R \& D$ than firms in West Germany do. In total, there is no empirical evidence for an innovation and R\&D lack at the firm level.
\end{abstract}

\section{Zusammenfassung}

Dieser Beitrag untersucht die Innovationsstrategien und Forschungs- und Entwicklungsaktivitäten (FuE) ostdeutscher Unternehmen sowie mögliche Innovationsdefizite in diesem Zusammenhang. Als Hinweis auf die zu geringen Innovationsaktivitäten ostdeutscher Unternehmen werden häufig die niedrigen FuE-Aufwendungen der Wirtschaft in den neuen Bundesländern angesehen. Doch die geringe FuE-Intensität auf aggregierter Ebene spiegelt lediglich die nachteilige ostdeutsche Wirtschafts- und Unternehmensgrößenstruktur wider. Eine Untersuchung des Innovations- und FuEVerhaltens deutscher Unternehmen auf der Basis des Mannheimer Innovationspanels zeigt, daß bei Berücksichtigung der ostdeutschen Wirtschaftsund Unternehmensgrößenstrukturen die Unternehmen im Verarbeitenden Gewerbe Ostdeutschlands häufiger und mit einer höheren Intensität FuE durchführen als (vergleichbare) westdeutsche Unternehmen. Auch bei anderen Innovationsindikatoren, wie z.B. dem Anteil der Unternehmen mit Basis- oder Verbesserungsinnovationen oder der Höhe der über FuE hin- 
ausgehenden Innovationsaufwendungen, läßt sich auf der Unternehmensebene kein Rückstand der ostdeutschen Unternehmen feststellen. 


\section{Ausgangsüberlegungen}

War der Beginn der Revitalisierung der ostdeutschen Industrie gekennzeichnet von einem kräftigen Wachstumsprozeß und dynamischen Investitionstätigkeiten, gibt es nun erste Hinweise auf ein Nachlassen des Aufschwungtempos. Es gibt Anzeichen dafür, daß es sich nicht nur um eine konjunkturelle Atempause handelt, sondern um eine längerfristige Schwäche. Bei den meisten gesamtwirtschaftlich relevanten Kennziffern, wie z.B. dem Pro-Kopf-Einkommen, verläuft die Angleichung an Westdeutschland langsam, und ein Abbau der Arbeitslosigkeit ist nicht in Sicht.

In dieser Situation wirft die Suche nach dem zukünftigen Erfolgsrezept eine Reihe von Fragen auf. Unklar ist, wie ein sich selbst tragendes Wachstum auf den Schultern von wettbewerbsfähigen verarbeitenden und dienstleistenden Unternehmen geschaffen werden kann, welche Möglichkeiten die einzelnen Betriebe haben und mit welcher Strategie sie versuchen sollten, ihre Marktchancen zu verbessern.

Der industriellen Forschung und Entwicklung und dem Innovationsverhalten der Unternehmen werden in diesem Kontext für Ostdeutschland eine hohe Bedeutung beigemessen. Sie sollen den wirtschaftlichen Aufholprozeß vorantreiben. Aber die industrielle FuE in den neuen Bundesländern stagniert auf einem geringen Niveau. Daran haben auch die erheblichen Anstrengungen der Forschungs- und Technologiepolitik nur insofern etwas geändert, als ohne diese Aktivitäten die Industrieforschung Ost wahrscheinlich noch stärker zurückgegangen wäre. Der Anteil Ostdeutschlands an den Gesamtaufwendungen für FuE im Verarbeitenden Gewerbe der Bundesrepublik ist jedoch weiterhin verschwindend gering. Er lag in den Jahren 1992 bis 1994 unter 5 Prozent (siehe die Tabellen 3 und 4 im Anhang). Die geringen FuE-Aufwendungen der ostdeutschen Wirtschaft auf aggregierter Ebene spiegeln jedoch lediglich die nachteiligen Wirtschaftsund Größenstrukturen wider und sind noch kein Hinweis für Innovationsdefizite auf der Unternehmensebene.

Auf die Diskussion dieser Befunde wird im ersten Teil des Beitrags eingegangen. Es wird gezeigt, daß bei anderen Innovationsindikatoren, wie z.B. dem Anteil der Unternehmen mit Innovationen oder der Höhe der über FuE hinausgehenden Innovationsaufwendungen, sich kein Rückstand feststellen läßt. Im Gegenteil: Hinsichtlich der FuE zeigt sich, daß bei Berücksichtigung der Wirtschafts- und Unternehmensgrößenstrukturen die ost- 
deutschen Unternehmen im Verarbeitenden Gewerbe häufiger und mit einer höheren Intensität FuE durchführen als (vergleichbare) westdeutsche Unternehmen.

Während dem Rückstand der ostdeutschen FuE auf aggregierter Ebene große Aufmerksamkeit geschenkt wird, wird weniger in Frage gestellt, welche Bedeutung FuE für das einzelne ostdeutsche Unternehmen in der jetzigen Situation des Aufholens hat. Industrielle Forschung und Entwicklung sind unternehmerische Entscheidungen und können nur im Kontext des wirtschaftlichen Entwicklungsstadiums des (der) Unternehmen(s), eines Wirtschaftszweiges, einer Region oder anderer Bezugsgrößen bewertet werden. Diesem Aspekt wird im zweiten Teil des vorliegenden Beitrags besondere Aufmerksamkeit geschenkt. Im Mittelpunkt der Überlegungen steht dabei die Absicht, auf der Datenbasis des Mannheimer Innovationspanels zu zeigen, daß eine Reihe von ostdeutschen Unternehmen erfolgreich innoviert und wirtschaftet, ohne daß der eigenen FuE von Anfang an ein großer Stellenwert zukommt - und umgekehrt, daß es vielen ostdeutschen Unternehmen, die von Anfang an hohe FuE-Intensitäten aufweisen, nicht gelungen ist, in ausreichendem Maße wirtschaftlich leistungsfähig zu werden.

\section{Strukturelle Gegebenheiten in der ostdeutschen Wirtschaft}

Betrachtet man die strukturellen Gegebenheiten in der ostdeutschen Wirtschaft, kann die niedrige FuE-Intensität nicht verwundern: FuE-Aktivitäten finden vor allem in Unternehmen des Verarbeitenden Gewerbes statt. Nach dem drastischen Rückgang der Industriebeschäftigten auf ca. eine Million erreicht der Beschäftigtenanteil des ostdeutschen Verarbeitenden Gewerbe an allen Beschäftigten in Ostdeutschland nicht einmal 60 Prozent der entsprechenden Relation in Westdeutschland.

Eine weitere wichtige Ursache für die geringe ostdeutsche FuE-Intensität ist in dem Fehlen von Großunternehmen zu sehen, die nahezu immer FuEAktivitäten aufweisen. In den Großunternehmen mit mehr als 500 Beschäftigten in Westdeutschland sind fast 50 Prozent der Werktätigen beschäftigt, in den vergleichbaren Unternehmen in den neuen Ländern sind es noch nicht einmal 25 Prozent. Genau umgekehrt ist das Verhältnis bei den Unternehmen mit weniger als 20 Beschäftigten. Hier finden sich in Ostdeutschland über 40 Prozent der Beschäftigten, während in den alten Ländern nur 20 Prozent der Arbeitnehmer zu dieser Größenklasse gehören. 
Kleine und mittlere Unternehmen (KMU) mit weniger als 500 Beschäftigten weisen zwar in den meisten Branchen, sofern sie FuE durchführen, ähnlich hohe FuE-Intensitäten auf wie die Großunternehmen, doch viele kleine und mittelständische Unternehmen verzichten auf FuE.

Desweiteren wiegt in Ostdeutschland das Fehlen oder zumindest die bisher schleppende Entwicklung potentiell forschungsintensiver Wirtschaftszweige innerhalb des Verarbeitenden Gewerbes schwer. Im bisherigen Verlauf des 'Aufbaus Ost' sind vor allem die zukunftswichtigen innovations- und FuE-intensiven Wirtschaftszweige wie die Elektrotechnik, die chemische Industrie, der Maschinen- und Fahrzeugbau, gemessen an der Beschäftigten- und Umsatzentwicklung, der Investitionstätigkeit oder den Exporten, zurückgeblieben.

Die Auflistung dieser Befunde macht deutlich, daß zur Referenzgröße 'Westdeutsche Industrie' der Anteil des FuE-Personals in ostdeutschen verarbeitenden Unternehmen aufgrund der ostdeutschen Industriestrukturen fast 'natürlicherweise' deutlich unter dem westdeutschen Niveau liegt. Entsprechend ist das gesamte FuE-Aufkommen in den neuen Ländern deshalb so niedrig, weil es kaum noch Großunternehmen mehr gibt, die Betriebe $(\mathrm{zu})$ klein und forschungsintensive Wirtschaftszweige in den neuen Bundesländern unterbesetzt sind.

\section{FuE und Innovationen in ostdeutschen Unternehmen}

\subsection{Kenngrößen zum Innovationsverhalten}

FuE ist lediglich ein Indikator für das Erfassen des Innovationsgeschehens. Er wird häufig auch als die 'Spitze des Eisbergs' bezeichnet. Zieht man weitere Indikatoren heran, um die Innovationsaktivitäten in den Unternehmen zu messen, so lassen sich zumindest auf den ersten Blick keine Innovationsdefizite in Ostdeutschland festmachen. Die Untersuchungen des ZEW zeigen, daß die ostdeutschen Unternehmen sehr stark in Innovationsprozesse eingebunden sind. Im Verarbeitenden Gewerbe weisen rund die Hälfte der ostdeutschen Unternehmen Produkt- oder Prozeßinnovationen auf und der Anteil der FuE betreibenden Unternehmen ist seit 1992 leicht gestiegen und liegt im Jahr 1994 bei 35 Prozent (siehe Tabelle 3 im Anhang). Hinsichtlich der Anteile von Unternehmen mit Produkt- und 
Prozeßinnovatoren bestehen zwischen dem ost- und westdeutschen Verarbeitenden Gewerbe sehr geringe Unterschiede.1 Zwischen 1990 und 1992 haben sich viele ostdeutsche Innovatoren entweder nur auf Produkt- bzw. nur auf Prozeßinnovationen konzentriert. 60 Prozent der ostdeutschen Innovatoren führten sowohl Produkt- als auch Prozeßinnovationen durch. Dieser Anteil ist im Jahr 1994 auf 80 Prozent gestiegen und entspricht etwa dem westdeutschen Anteil.

Wie bedeutend die Produktinnovationen für die ostdeutschen Unternehmen waren, läßt sich darin erkennen, daß im Jahr 1994 rund 46 Prozent des erzielten Umsatzes im ostdeutschen Verarbeitenden Gewerbe mit Produkten erwirtschaftet wurde, die im Zeitraum von 1992 bis 1994 neu in das Produktsortiment der Unternehmen aufgenommen bzw. verbessert wurden. In Westdeutschland liegt der Umsatzanteil mit neuen oder verbesserten Produkten im Verarbeitenden Gewerbe bei 41 Prozent.

In der technologiepolitischen Diskussion über das (ost-)deutsche Innovationsgeschehen konzentriert man sich häufig zu sehr auf die FuE, das heißt man betrachtet lediglich einen Aspekt des Innovationsgeschehens. Aber nicht alle Unternehmen, die neue Produkte oder Herstellungsverfahren auf den Markt bringen, forschen. Zwar führen 70 Prozent der ostdeutschen Innovatoren bzw. 74 Prozent der westdeutschen Innovatoren im Verarbeitenden Gewerbe FuE durch. Aber die Auswertungen des Mannheimer Innovationspanel zeigen auch, daß bei den Unternehmen mit FuE ein großer Teil der gesamten Innovationsaufwendungen nicht auf FuE entfällt. Dies gilt insbesondere für Ostdeutschland. Im Jahr 1992 entfielen rund 80 Prozent der Innovationsaufwendungen auf Investitionen für Innovationsprojekte. Bedingt durch den Aufhol- und Transformationsprozeß der ostdeutschen Wirtschaft waren 1992 nahezu alle Investitionsausgaben der Unternehmen Investitionen in den Innovationsprozeß. Der veraltete Kapitalstock wurde ausgetauscht bzw. für das erneuerte Produktsortiment wurden neue Maschinen und Ausrüstungsgüter benötigt. Durch die sehr hohen Investitionen für Innovationen im Jahr 1992 betrug das Verhältnis der Innovationsaufwendungen zum Umsatz im ostdeutschen Gewerbe 15,9 Prozent, während diese Relation in Westdeutschland nur bei 4,7 Prozent lag. Diese Phase der Modernisierung des Kapitalstocks war nach 1992 weitgehend abgeschlossen. Die weiterhin hohen Investitionsaufwendungen sind nun zu einem größeren Teil Erweiterungsinvestitionen. Die Investitionen für In-

1 Vergleiche hierzu und im folgenden die Tabellen 3 und 4 im Anhang. 
novationen gingen dagegen sehr stark zurück, so daß das Verhältnis der Innovationsaufwendungen zum (in der Zwischenzeit gestiegenen) Umsatz im Jahr 1994 knapp 7 Prozent betrug.

Aber auch 1994 macht die eigentliche Forschung und experimentelle Entwicklung (inklusive den investiven Aufwendungen im FuE-Bereich wie z.B. für Laborgeräte) lediglich ein Drittel der gesamten ostdeutschen Innovationsaufwendungen aus, während im westdeutschen Verarbeitenden Gewerbe dieser Anteil bei zwei Drittel liegt.2 Betrachtet man die gesamten FuE-Aufwendungen in Relation zum gesamten Umsatz, erreicht das ostdeutsche Verarbeitende Gewerbe eine Relation von 2,1 Prozent, das westdeutsche Verarbeitende Gewerbe 2,9 Prozent. 3

Zusammenfassend läßt sich sagen, daß in Ostdeutschland durchaus Innovationen stattfinden und zum Teil die Innovationsintensität in den Unternehmen über dem westdeutschen Niveau liegt. Offen ist jedoch, warum der relativ hohe Innovationsinput wie auch der hohe Innovationsoutput (z.B. Umsatzanteile mit neuen und verbesserten Produkten) nicht zu einem gröBeren Umsatz- und Beschäftigungswachstum des ostdeutschen Verarbeitenden Gewerbes geführt haben. Gibt es doch ein Innovationsdefizit, das sich eher in der Wahl der Innovationsstrategie auf der Unternehmensebene widerspiegelt und weniger in der Höhe der aggregierten FuE- und Innovationsaufwendungen zum Ausdruck kommt?

\subsection{Innovationsstrategien und Kapitalverflechtungen}

Die Bedeutung von industrieller Forschung und Entwicklung für ein Land bzw. für ein einzelnes Unternehmen sollte, wie gesagt, im Kontext seines wirtschaftlichen Entwicklungsstadiums gesehen werden. Wachstumsmöglichkeiten und internationale Wettbewerbsfähigkeit sind Ausdruck des zum jeweiligen Zeitpunkt effizienten Einsatzes der verfügbaren Ressourcen;

2 Die gesamten FuE-Aufwendungen und Innovationsaufwendungen im westdeutschen Verarbeitenden Gewerbe werden vorwiegend von den Großunternehmen bestimmt. Bei den kleinen und mittleren Unternehmen in Westdeutschland ist der Anteil der FuE-Aufwendungen an den gesamten Innovationsaufwendungen wesentlich geringer.

3 Die relativ hohe Relation FuE-Aufwendungen/Umsatz könnte auch an dem sehr niedrigen ostdeutschen Umsatzniveau liegen. Um dieser Interpretation $\mathrm{zu}$ begegnen, wird dort die Relation FuEBeschäftigte zu Gesamtbeschäftigten betrachtet. Im dritten Abschnitt wird aufgezeigt, daß unter Berücksichtigung der erwähnten ungünstigen Strukturmerkmale im Verarbeitenden Gewerbe die FuEIntensität als hoch einzuschätzen ist. 
dies gilt auch für den Faktor FuE. Gittlemann und Wolff (1995) zeigen, daß nur in hochentwickelten Ländern Forschung und Entwicklung einen signifikanten Beitrag zum Wirtschaftswachstum leistet. Für aufholende Länder, die noch weit von der Produktivität der führenden Länder entfernt sind, wird FuE zum einen in geringerem Maße eingesetzt und zum anderen trägt dieser Einsatz nicht signifikant zum Wirtschaftswachstum bei. Technischer Fortschritt spielt auch bei diesen Ländern eine große Rolle. ${ }^{4}$ Bei den aufholenden Ländern entsteht jedoch dieser technischer Fortschritt durch Imitation und Adaption von Technologien aus technologisch führenden Ländern, insbesondere durch Kauf von Ausrüstungsgütern sowie dem anschließenden Lernen durch den Umgang und Gebrauch neuer Produkte und Prozesse (vgl. Grossman und Helpman, 1993 und Felder, 1998).

Vor diesem Hintergrund müssen auch die FuE- und Innovationsaktivitäten der ostdeutschen Unternehmen gesehen werden bzw. es sollte beleuchtet werden, warum sich ein Teil der ostdeutschen Unternehmen nicht entsprechend dem 'Muster' technologisch aufholender Unternehmen verhalten. In stilisierter Form lassen sich zwei Innovationsstrategien konstatieren, die in den vergangenen Jahren bei den ostdeutschen Unternehmen zu beobachten waren: Ein Teil der Unternehmen hat aufgrund des schnelleren und leichteren Zugriffs auf westliche Technologien und Kapital sowie durch den Wettbewerbsdruck die reine investitionsgeleitete (Technologienehmer-) Phase sehr schnell durchlaufen. Für diese Gruppe spielten eigene FuEAktivitäten und eine eigene Produktentwicklung am Beginn des Aufholprozesses eine geringere Bedeutung. Mit zunehmender Angleichung an die wirtschaftliche Leistungsfähigkeit westdeutscher Unternehmen werden in absehbarer Zeit viele dieser ostdeutschen Unternehmen in die nächste Aufholphase wechseln bzw. sie haben es bereits heute getan. Die eigene FuE und die eigene Produktentwicklung wird in dieser zweiten Phase verstärkt an Bedeutung gewinnen.

Es sind aber auch Unternehmen zu beobachten, die aus verschiedenen Gründen, wie beispielsweise aufgrund des starken Wettbewerbs, den geringen Kostenvorteilen gegenüber westdeutschen Unternehmen sowie die

4 Unter technischem Fortschritt wird der Beitrag zum Wirtschaftswachstum verstanden, der nicht durch den Mehreinsatz der Faktoren Arbeit, Kapital und gegebenenfalls Material/Rohstoffe erklärt werden kann. Während in den hochindustrialisierten Ländern der Faktor ,technischer Fortschritt“ nahezu 50 Prozent zum Wirtschaftswachstum beiträgt, liegt dieser Anteil bei den noch nicht entwickelten Ländern bei einem Drittel (vgl. Gittleman und Wolff, 1995). Die Zunahme des Pro-Kopf-Einkommens wird bei diesen Ländern vorwiegend von der Zunahme der Kapitalintensität bestimmt. 
für sie vorliegenden eingeschränkten Möglichkeiten des Technologietransfers, nicht die Rolle eines Technologienehmers einnahmen und von Beginn des Aufholprozesses an eigene Forschungsanstrengungen in den Mittelpunkt ihrer Innovationsstrategie gestellt haben. Überspitzt formuliert, läßt sich diese Strategie oder besser dieser Versuch als 'Überholen mit Hilfe von FuE ohne Aufzuholen' beschreiben.

Für die Wahl der Innovationsstrategie spielt in diesem Zusammenhang die Kapitalverflechtung, d.h. die Zugehörigkeit zu einem westdeutschen oder ausländischen Unternehmen(-sverbund) eine wichtige Rolle.5 Erfolgreiche Wettbewerbs- und Innovationsstrategien können darauf zurückgeführt werden, daß den Unternehmen eine Integration in das (west)deutsche Innovationssystem gelungen ist. Diese Einbindung gelang offensichtlich ostdeutschen Unternehmen, die mit westdeutschen Unternehmen über eine Kapitalverflechtung verbunden sind, weitaus besser. Mögliche Ursache für die Wahl unterschiedlicher Innovationsstrategien von unabhängigen und zu einer Unternehmensgruppe gehörenden ostdeutschen Unternehmen ist, daß für Unternehmen in einem Verbund bestimmte Restriktionen in geringerem Umfang zutreffen und einige Möglichkeiten besser genutzt werden können. Unter anderem zeigen sich für verbundene Unternehmen im Vergleich zu unabhängigen folgende Vorteile:

- geringere Finanzierungsrestriktionen und daher

- mehr Investitionsmöglichkeiten

- Nutzung des Technologietransfers innerhalb der Unternehmensgruppe

- Austausch von Managementwissen und Humankapital

- bessere Arbeitsteilung und höherer Rationalisierungsdruck

- Nutzung von Vertriebsnetzen und besserer Zugang zu (inter)nationalen Märkten.

In Ostdeutschland gehören zwischen 20 und 30 Prozent der Unternehmen aus dem Verarbeitenden Gewerbe zu einer westdeutschen oder ausländi-

5 Siehe hierzu und im folgenden Barjak, Felder und Fier (1998). 
schen Unternehmensgruppe. 6 Größere Unternehmen befinden sich weitaus häufiger im Westbesitz. In bezug auf das Innovationsverhalten lassen sich die Westtöchter dahingehend charakterisieren, daß sie zu Beginn des Aufholprozesses Technologienehmer waren, die von ihrer Muttergesellschaft häufig die Produkte und die Produktionsverfahren übernahmen und über hohe Investitionsaktivitäten ihren Kapitalstock modernisierten. Die unabhängigen ostdeutschen Unternehmen weisen geringere Investitionsaktiväten auf. Dafür haben die unabhängigen ostdeutschen Unternehmen im Vergleich zu den Westtöchtern wesentlich höhere FuE-Aktivitäten. Mit zum Teil FuE-Personalintensitäten von weit über zehn Prozent hat sich eine Verzerrung des Verhältnisses FuE-Input zu Produktion und zu wirtschaftlichem Erfolg ergeben. Nur wenige Unternehmen haben mit der Strategie des 'Überholens mit Hilfe von FuE' Erfolg gehabt. Häufig sind dabei durchaus technische Erfolge vorzuweisen, aber ein großer Teil der Marktneuheiten konnte keinen ausreichenden Markt(anteil) und Umsatz auf sich ziehen.

Dabei darf nicht übersehen werden, daß etliche Unternehmen bei der Bewältigung der dramatischen Umbrüche in der ostdeutschen Wirtschaft nur einen begrenzten Handlungsspielraum hatten. Naheliegend ist, daß sie zunächst versucht haben, auf der Basis ihres eigenen Know-hows und ihrer bisherigen wissenschaftlichen Tätigkeit den Anpassungsprozeß zu schaffen. Die Unternehmen wollten sich aus eigener Kraft und im Rahmen ihrer Möglichkeiten Märkte erschließen. Sie mußten erkennen, daß die Innovationskraft eines Unternehmens sich dabei nicht nur auf die technische Seite erstreckt, sondern auch auf die Fähigkeit, originelle Ideen in marktfähige Produkte umzusetzen.

In den ökonometrischen Analysen, kontrolliert nach Wirtschaftszweigzugehörigkeit und Unternehmensgröße, läßt sich feststellen, daß 1994 ostdeutsche Tochtergesellschaften eine um 23 Prozent höhere Arbeitsproduktivität (Umsatz/Beschäftigte) aufweisen, als unabhängige ostdeutsche Unternehmen. Es läßt sich auch zeigen, daß diese Differenz nicht nur auf eine

6 Basis dieser Segmentation sind ca. 1.800 ostdeutsche Unternehmen des Verarbeitenden Gewerbes, die zwischen 1993 und 1995 an einer der Befragungen des Mannheimer Innovationspanels teilnahmen und nach dem Kriterium Zugehörigkeit zu einer Unternehmensgruppe aufgeteilt wurden. 30 Prozent der ostdeutschen Unternehmen gehörten in diesem Sample zu einer westdeutschen oder ausländischen Unternehmensgruppe. Hochgerechnet auf das gesamte ostdeutsche Verarbeitende Gewerbe kann man davon ausgehen, daß sich mindestens 20 Prozent der Unternehmen im Westbesitz befinden, auf die mindestens ein Drittel der Beschäftigten entfällt. 
positive Selektion von Unternehmen, die zu Westtöchtern wurden, zurückzuführen ist, sondern auch auf ein unterschiedliches Innovationsverhalten. Insbesondere die hohen Investitionsaufwendungen pro Beschäftigten der Westtöchter, die nahezu doppelt so hoch waren als bei den unabhängigen Firmen, erklären die unterschiedliche Produktivität.

\subsection{Mikroökonometrische Analyse des Innovationsverhaltens und der FuE-Aktivitäten}

In diesem Abschnitt werden drei zentrale Hypothesen des Beitrags auf der Basis des Mannheimer Innovationspanels für das Jahr 1994 überprüft:

a) Kontrolliert nach Wirtschaftszweigzugehörigkeit und Unternehmensgröße führen ostdeutsche Unternehmen im Vergleich zu westdeutschen Unternehmen nicht seltener FuE durch.

b) Auch hinsichtlich der FuE-Intensität liegen ostdeutsche FuE durchführende Unternehmen bei Berücksichtigung der beiden Strukturvariablen Wirtschaftszweigzugehörigkeit und Unternehmensgröße nicht zurück.

c) In Ostdeutschland führen viele Unternehmen FuE durch, ohne daß zur Zeit eine entsprechende wirtschaftliche Basis oder Leistungsfähigkeit vorhanden ist.

\section{Zu (a): FuE-Wahrscheinlichkeit}

Mit Hilfe linearer Wahrscheinlichkeitsmodelle (Probit-Modelle) wird geprüft, ob ostdeutsche Unternehmen unter Berücksichtigung ihrer Unternehmensgröße und ihrer Wirtschaftszugehörigkeit seltener oder häufiger FuE durchführen. In Tabelle 1 ist im linken Teil eine Schätzung für die Durchführung von FuE aufgeführt, wobei als erklärende Größen lediglich einige wenige Strukturgrößen verwendet werden. Bei dem verwendeten (speziellen) Probit-Modell können die Koeffizienten bei den DummyVariablen“7 als Änderung der Wahrscheinlichkeit interpretiert werden, wenn für ein Unternehmen - im Vergleich zu den Unternehmen der 'Referenzgruppe' - ein Sachverhalt zutrifft.

7 Eine Dummy-Variable hat den Wert null, wenn ein Tatbestand nicht zutrifft (Referenz) und den Wert eins wenn der Tatbestand zutrifft. 
Tabelle 1: FuE-Aktivitäten und FuE-Personalintensitäten im Verarbeitenden Gewerbe im Ost-Westvergleich (1992 bis 1994)

\begin{tabular}{|c|c|c|c|c|}
\hline & \multicolumn{2}{|c|}{$\begin{array}{l}\text { Schätzung der Durchfüh- } \\
\text { rung von FuE }\end{array}$} & \multicolumn{2}{|c|}{$\begin{array}{c}\text { Schätzung der FuE-Personal- } \\
\text { intensität bei Unternehmen mit } \\
\text { FuE }\end{array}$} \\
\hline Anzahl der Beobachtungen & \multicolumn{2}{|l|}{2.449} & \multicolumn{2}{|l|}{1.172} \\
\hline \multirow[t]{2}{*}{ Pseudo $\mathrm{R}^{2}$} & \multicolumn{2}{|l|}{0,16} & \multicolumn{2}{|l|}{0,33} \\
\hline & Koeffizient & t-Wert & Koeffizient & $\mathrm{t}$-Wert \\
\hline \multicolumn{5}{|l|}{ West- /Ostdeutschland } \\
\hline Westdeutsche Unternehmen & Referenz & & Referenz & \\
\hline Ostdeutsche Unternehmen & $0,09 * * *$ & 3,53 & $\mid 0,17 * *$ & 2,51 \\
\hline \multicolumn{5}{|l|}{ Unternehmensgröße: } \\
\hline 5- 49 Beschäftigte & Referenz & & Referenz & \\
\hline 50-249 Beschäftigte & $0,27 * * *$ & 10,59 & $-0,93 * * *$ & $-13,51$ \\
\hline$>=250$ Beschäftigte & $0,49 * * *$ & 18,83 & $\mid-1,27 * * *$ & $-16,39$ \\
\hline \multicolumn{5}{|l|}{ Wirtschaftszweige: } \\
\hline Holz, Papier, Druck & Referenz & & Referenz & \\
\hline Ernährung, Textil usw. & $-0,03$ & $-0,79$ & $-0,26^{* *}$ & $-2,21$ \\
\hline Chemie, Mineralölverarbeitung & $0,25 * * *$ & 5,43 & $0,78 * * *$ & 5,98 \\
\hline Kunststoffe, Gummi & $0,14^{* *}$ & 2,94 & $0,25 * *$ & 2,19 \\
\hline Glas, Keramik & 0,07 & 1,18 & $-0,05$ & $-0,33$ \\
\hline Metallerzeugung & 0,09 & 1,40 & $-0,37 * *$ & $-2,32$ \\
\hline Stahl- und Leichtmetallbau & 0,06 & 1,43 & $-0,21 *$ & $-1,84$ \\
\hline Maschinenbau & $0,29 * * *$ & 7,59 & $0,42 * * *$ & 4,25 \\
\hline ADV, Elektrotechnik & $0,30 * * *$ & 6,71 & $0,77 * * *$ & 6,82 \\
\hline Medizin-, Regelungstechnik & $0,31 * * *$ & 6,77 & $0,80 * * *$ & 6,92 \\
\hline Fahrzeugbau & $0,13^{* *}$ & 2,58 & $0,40 * * *$ & 2,86 \\
\hline \multicolumn{5}{|l|}{ FuE-Förderung: } \\
\hline keine FuE-Förderung & & & Referenz & \\
\hline öffentliche FuE-Förderung & & & $0,28 * * *$ & 4,80 \\
\hline Konstante & & & $-2656,42 * * *$ & $-25,79$ \\
\hline
\end{tabular}

Quelle: ZEW (1997) Unternehmenspanel Ost, Mannheimer Innovationspanel 
Das Probit-Modell zeigt, daß nach Kontrolle der Wirtschaftszweigzugehörigkeit und Unternehmensgröße ostdeutsche Unternehmen eine um neun Prozent höhere Wahrscheinlichkeit für die Durchführung von FuEAktivitäten aufweisen als ein (vergleichbares) westdeutsches Unternehmen. 8

\section{Zu (b): FuE-Intensität}

Die hochgerechneten Ergebnisse des Mannheimer Innovationspanels für das Jahr 1994 zeigen, daß westdeutsche FuE-durchführende Unternehmen des Verarbeitenden Gewerbes im Durchschnitt 8 Prozent ihres Personals für FuE einsetzen, während dieser Anteil bei den ostdeutschen FuEdurchführenden Unternehmen mit ca. 10,1 Prozent um rund 25 Prozent höher liegt.

Wie unterscheiden sich die FuE-Personalintensitäten beider Regionen, wenn nach den zwischen Ost- und Westdeutschland bestehenden Strukturunterschieden kontrolliert wird? Im rechten Teil von Tabelle 1 sind die Ergebnisse einer (heteroskedastierobusten) linearen Regression zur Erklärung der FuE-Personalintensität bei FuE-betreibenden Unternehmen aufgeführt. Für die Regression werden die logarithmierten FuE-Personalintensitäten9 verwendet.10 In der Regression werden die gleichen Einflußgrößen wie in der ersten Schätzung sowie zusätzlich eine Dummy-Variable für die Teilnahme an einem öffentlichen FuE-Programm in der Zeit zwi-

8 Die Schätzung macht auch deutlich, warum auf 'aggregierter Ebene' ostdeutsche Unternehmen dennoch seltener FuE durchführen: Unternehmen zwischen 50 und 250 Beschäftigten haben eine um 27 Prozent und Unternehmen über 250 Beschäftigten eine um 49 Prozent höhere Wahrscheinlichkeit für die Durchführung von FuE als Unternehmen mit weniger als 50 Beschäftigten. In Ostdeutschland sind aber im Vergleich zu Westdeutschland große Unternehmen unter- und Unternehmen mit weniger als 50 Beschäftigten überrepräsentiert.

9 Es wurden nur FuE-Personalintensitäten berücksichtigt, die größer als Null und kleiner als 0,6 waren. Da nur wenig Unternehmen solche (unrealistischen) oberen Extremwerte aufweisen ändern sich die Ergebnisse kaum, wenn man die Obergrenze für FuE-Personalintensitäten z.B. auf 0,4 setzt.

10 Mit 100 multipliziert können die in der Tabelle ausgewiesenen Koeffizienten der Dummy-Variablen approximativ als geschätzter prozentualer Unterschied bei der FuE-Personalintensität zwischen einem Unternehmen, das den Wert null und einem Unternehmen, das den Wert eins bei einer DummyVariable aufweist, betrachtet werden. Für Koeffizientenwerte, die betragsmäßig größer als 0,2 sind, wird die Approximation ungenauer. Die genaue Berechnung für prozentuale Änderungen zwischen den zwei Dummy-Werten null und eins lautet für einen vorliegenden Koeffizienten ß: prozentuale Änderung $=\left(e^{B}-1\right) \cdot 100$. 
schen 1992 und 1994 sowie eine Konstante verwendet. Für mittlere und große Unternehmen ergeben sich aus der Regression eine um 60 bzw. 70 Prozent niedrigere FuE-Personalintensität als bei Unternehmen mit 5 bis 50 Beschäftigten. Da Ostdeutschland einen wesentlich höheren Anteil an Unternehmen mit weniger als 50 Beschäftigten aufweist, erklärt dies zu einem Teil die auf deskriptiver Basis bestehenden Unterschiede zwischen Ost- und Westdeutschland. Verstärkend kommt hinzu, daß für Unternehmen, die zwischen 1992 und 1994 an FuE-Förderprogrammen teilgenommen haben, eine um über 32 Prozent höhere FuE-Personalintensität geschätzt wird. Der Anteil bei den FuE betreibenden Unternehmen, die FuEFörderung erhalten, ist in Ostdeutschland mehr als doppelt so hoch als in Westdeutschland. Dennoch zeigt sich, daß auch nach Kontrolle für Unternehmensgröße, Wirtschaftszweigzugehörigkeit und FuE-Förderung ostdeutsche Unternehmen mit FuE immer noch eine um 17 Prozent höhere FuE-Personalintensität aufweisen. Viele ostdeutsche Unternehmen setzen auf eine sehr intensive FuE-Strategie.

Die Ergebnisse aus den beiden Schätzungen in Verbindung mit den FuEKennziffern auf aggregierter Ebene lassen sich folgendermaßen zusammenfassen: Die ostdeutsche Wirtschaft hat an sich kein 'FuE-Problem'. Berücksichtigt man die für FuE-Aktivitäten ungünstigen Wirtschafts- und Größenstrukturen in Ostdeutschland, führen im Vergleich zu Westdeutschland eher mehr Unternehmen FuE durch und insbesondere betreiben diese ostdeutschen Unternehmen FuE mit einer vergleichsweise sehr hohen Intensität. Das niedrige ostdeutsche FuE-Aufkommen ist ausschließlich eine Folge der Wirtschafts- und Unternehmensgrößenstruktur.

\section{Zu (c): Arbeitsproduktivität und FuE}

Wie vorher angesprochen, kann man davon ausgehen, daß FuE erst ein wichtiger Faktor für das Produktivitätswachstum für Unternehmen wird, wenn sie sich der 'weltweiten Produktionsmöglichkeitskurve' (production frontier) angenähert haben (vgl. Fäere et al., 1994; Felder, 1998). Dies ist zum einen darauf zurückzuführen, daß von einem 'gewissen' Grad der Annäherung

- für ein weiteres Aufholen durch Imitation und Adaption von Technologien eigene FuE-Aktivitäten notwendig werden und

- die Möglichkeit, durch eigene, originäre Innovationen die 'weltweite 
Produktionsmöglichkeitskurve' zu verschieben, größer wird.

Nur in dem seltenen Fall der Entwicklung völlig neuer Technologien, bei denen altes technologisches Wissen nicht von großem Nutzen ist, wäre ein sofortiger technologischer 'Sprung' mit Hilfe von FuE vorstellbar. Lediglich für wenige 'zurückliegende' Unternehmen dürfte es daher möglich oder effizient sein, den Prozeß des Aufholens auszulassen und durch eigene FuE eine neue Effizienzkurve zu generieren. Selbst wenn es dem technisch zunächst zurückliegenden Unternehmen gelingt, eine Marktneuheit zu entwickeln, besteht jedoch das Risiko, daß die neuen Produkte oder Prozesse nicht dem technischen Stand entsprechen, der FuE-Aufwand den Ertrag der Marktneuheit übersteigt oder die Innovation durch effizienter produzierende Unternehmen schnell übernommen wird und somit keine ausreichende Rentabilität erreicht werden kann.

Nach unserer Vermutung ist der geringe Erfolg der Innovationsaktivitäten in bezug auf Umsatz- und Beschäftigungswachstum darauf zurückzuführen, daß viele ostdeutsche Unternehmen FuE durchführen, ohne daß sie hierfür zur Zeit eine entsprechende wirtschaftliche Basis oder Leistungsfähigkeit aufweisen. In Tabelle 2 sind die Ergebnisse zweier ProbitSchätzungen aufgeführt, in denen ähnlich wie in Tabelle 1 jedoch getrennt für Ost- und Westdeutschland die Durchführung von FuE bei Unternehmen des Verarbeitenden Gewerbes geschätzt werden. Als weitere erklärende Variable für die momentane FuE-Entscheidung wird die Höhe der Arbeitsproduktivität (in logarithmierter Form ) als Indikator für die aktuelle wirtschaftliche Leistungsfähigkeit berücksichtigt.

Der fundamentale Unterschied zwischen Ost- und Westdeutschland liegt darin, daß in Westdeutschland die FuE-betreibenden Unternehmen eine um 13 Prozent höhere Arbeitsproduktivität aufweisen als nicht FuE-betreibende Unternehmen in Westdeutschland. Ostdeutsche Unternehmen mit FuE haben dagegen eine um 16 Prozent niedrigere Arbeitsproduktivität als ostdeutsche Unternehmen ohne FuE (siehe die Koeffizienten in Tabelle 2). 11 Dieser letzte Vergleich bezog sich wohlbemerkt auf die Unternehmen

11 Positiv anzumerken ist, daß schon im Jahr 1994 die Westtöchter genauso häufig FuE durchführen wie unabhängige ostdeutsche Unternehmen. Allerdings ist die FuE-Intensität etwa um die Hälfte geringer als bei den unabhängigen ostdeutschen Unternehmen, d.h. auf dem Niveau westdeutscher Unternehmen. 1992 war die Wahrscheinlichkeit für die Durchführung von FuE bei Westtöchtern noch um 15 Prozent geringer als bei den unabhängigen Unternehmen (vgl. auch Felder et al. , 1995) 
innerhalb Ostdeutschlands; der Produktivitätsabstand der ostdeutschen Unternehmen mit FuE zu westdeutschen Unternehmen beträgt ca. 50 Prozent.

Tabelle 2: Durchführung von FuE im Verarbeitenden Gewerbe - getrennt für Ost- und Westdeutschland im Jahr 1994

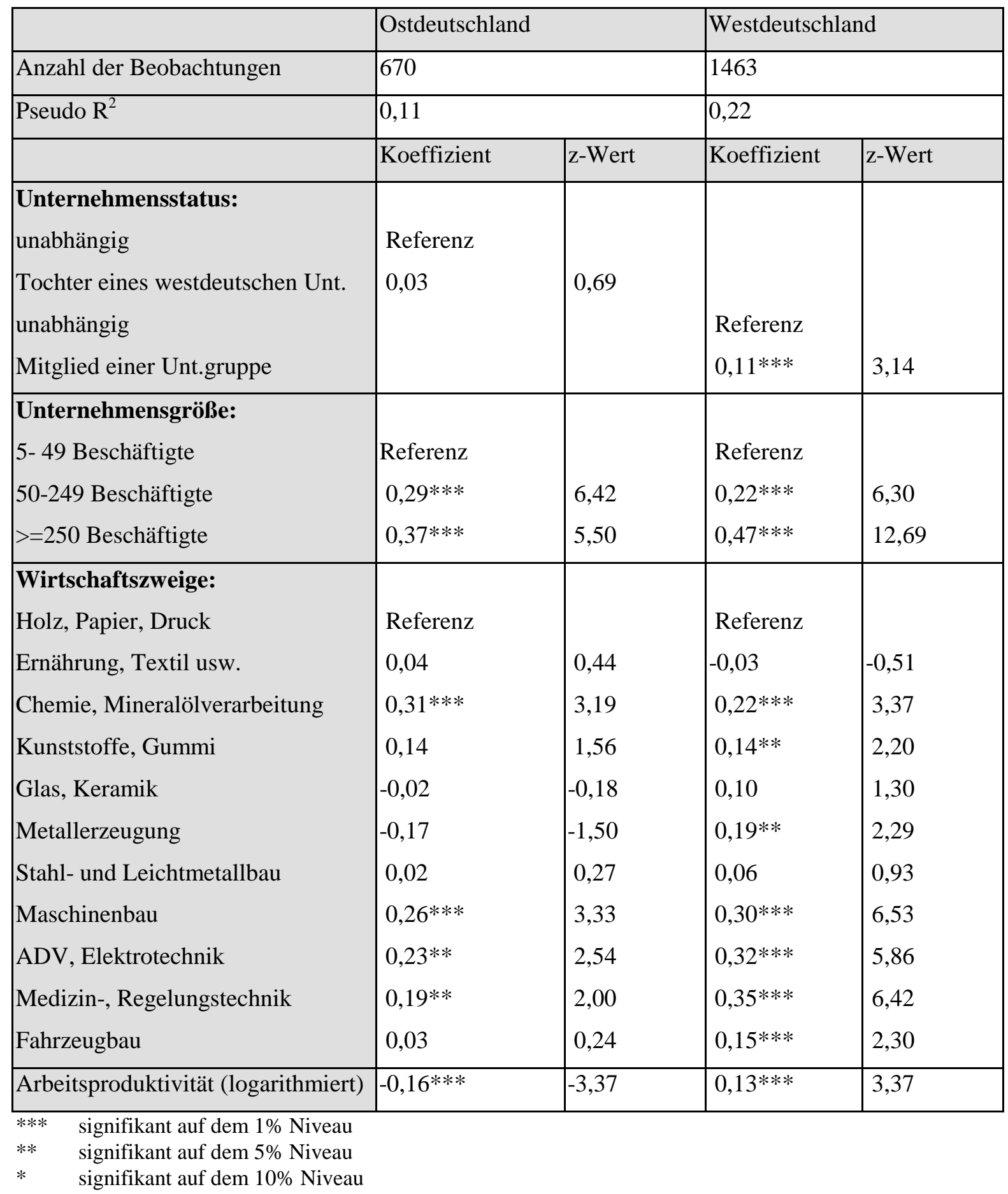

Quelle: ZEW (1997) Unternehmenspanel Ost, Mannheimer Innovationspanel 
Das Ergebnis sagt aber noch nicht, daß eine frühe FuE-Strategie nicht zu einem starken Anstieg der Arbeitsproduktivität geführt hat. Betrachtet man die Unternehmen, für die Daten auch aus dem Jahr 1992 oder 1993 aus dem Mannheimer Innovationspanel vorlagen, zeigt sich, daß ostdeutsche Unternehmen mit FuE im Jahr 1992 oder 1993 im Jahr 1994 eine um 10 Prozent niedrigere Arbeitsproduktivität aufweisen als ostdeutsche Unternehmen ohne FuE in diesen Jahren. Zwar stieg die Arbeitsproduktivität der ostdeutschen FuE-betreibenden Unternehmen zwischen 1992 und 1994 an, dies ist aber nahezu ausschließlich auf den Beschäftigungsabbau zurückzuführen. Erste Auswertungen für das Jahr 1995 und 1996 lassen nicht erkennen, daß ostdeutsche Unternehmen mit früher FuE-Strategie jetzt mit zeitlicher Verzögerung Markterfolge aufweisen.

\section{Fazit und abschließende Bemerkungen}

Betrachtet man eine Vielzahl von Innovationsindikatoren weisen die Unternehmen im Verarbeitenden Gewerbe Ostdeutschlands keinen Rückstand gegenüber westdeutschen Firmen. Lediglich bei der Betrachtung der aggregierten FuE-Aufwendungen bleibt Ostdeutschland zurück. Dies läßt sich ausschließlich durch den geringeren (Beschäftigungs- oder Wertschöpfungs-) Anteil FuE-intensiver Wirtschaftszweige in der ostdeutschen Industrie und insbesondere durch das Fehlen von Großunternehmen zu erklären ist. Im Gegenteil ist zu betonen, daß unter Berücksichtigung der strukturellen Gegebenheiten und des technologischen Rückstands in vielen Unternehmen die FuE überproportioniert ist. Am Beispiel ostdeutscher Tochterunternehmen mit einer westdeutschen oder ausländischen Mutter wird deutlich, daß eine Innovationsstrategie, die zunächst den Schwerpunkt auf westlichen Technologietransfer, hohe Investitionstätigkeiten und den Aufbau einer effizienten Produktion legte, erfolgreicher war. Diese Unternehmen beginnen nun in der zweiten Phase des Aufholprozesses mit FuE.

Dagegen haben viele ostdeutsche unabhängige Unternehmen, die von Anfang an eine FuE-Strategie unter Vernachlässigung anderer Innovationsaktivitäten verfolgten, nicht die wirtschaftlichen und technologischen Voraussetzungen für die erfolgreiche Umsetzung ihrer technischen FuEErgebnisse in Markterfolge. Eine wichtige, zukünftige technologiepolitische Herausforderung dürfte es daher sein, diesen Unternehmen beim Aufbau der wirtschaftlichen Voraussetzungen für erfolgreiche FuE zu helfen. 
Wichtig ist es, daß es diesen Unternehmen gelingt, eine effizienten Produktion aufzubauen, Kundenorientierung zur prioritären Aufgabe wird und sie in das gesamtdeutsche Innovationssystem eingebunden werden, damit zum einen der Zugang zu unternehmensexternem westlichem Wissen verbessert wird und zum anderen bestehende Vertriebswege genutzt werden können.

\section{Anhang}

Tabelle 3: Kenngrößen zum Innovationsverhalten im ostdeutschen Verarbeitenden Gewerbe

\begin{tabular}{|c|c|c|c|c|c|c|c|c|c|}
\hline & \multicolumn{3}{|c|}{$1994^{2)}$} & \multicolumn{3}{|c|}{1993} & \multicolumn{3}{|c|}{1992} \\
\hline & absolut & in & & absolut & in $c$ & & absolut & in & $\%$ \\
\hline $\begin{array}{l}\text { Unternehmen }^{1)} \\
\text { darunter: }\end{array}$ & 7100 & 100 & & 7400 & 100 & & 8500 & 100 & \\
\hline $\begin{array}{l}\text { Innovatoren } \\
\text { darunter: }\end{array}$ & 3600 & 51 & 100 & 3600 & 49 & 100 & 4200 & 50 & 100 \\
\hline Produktinnovatoren & 3300 & 46 & 92 & 3000 & 41 & 83 & 3300 & 39 & 79 \\
\hline Prozeßinnovatoren & 3100 & 43 & 86 & 3000 & 41 & 83 & 3300 & 39 & 79 \\
\hline FuE-Treibende & 2500 & 35 & 70 & 1900 & 26 & 53 & 2300 & 27 & 55 \\
\hline FuE-Abteilung & 1000 & 14 & 28 & 900 & 12 & 25 & 1100 & 13 & 26 \\
\hline $\begin{array}{l}\text { Umsatz (in Mrd. DM) } \\
\text { darunter: }\end{array}$ & 120 & 100 & & 112 & 100 & & 102 & 100 & \\
\hline $\begin{array}{l}\text { Innovatoren } \\
\text { darunter: }\end{array}$ & 90 & 74,9 & 100 & 80 & 71,7 & 100 & 74 & 72,4 & 100 \\
\hline Produktinnovatoren & 82 & 68,3 & 91,1 & 76 & 67,5 & 95,0 & 66 & 65,0 & 89,2 \\
\hline Prozeßinnovatoren & 83 & 69,3 & 92,2 & 69 & 61,6 & 86,3 & 59 & 57,7 & 79,7 \\
\hline $\begin{array}{l}\text { FuE-Treibende } \\
\text { nach Art der Produkte: }\end{array}$ & 72 & 60,1 & 80,0 & 48 & 42,5 & 60,0 & 55 & 53,9 & 74,3 \\
\hline neue oder wesentlich verbesserte & 33 & 27,5 & 36,7 & 34 & 30,4 & 42,5 & 23 & 22,5 & 31,1 \\
\hline verbesserte & 23 & 19,2 & 25,6 & 21 & 18,8 & 26,3 & 21 & 28,4 & 20,6 \\
\hline nicht/ nur unerheblich verbesserte ${ }^{3)}$ & 64 & 53,3 & $37,7^{*}$ & 57 & 50,9 & $31,2 *$ & 58 & 49,1 & $48,3 *$ \\
\hline $\begin{array}{l}\text { Innovationsaufwendungen (in Mrd. } \\
\text { DM) }\end{array}$ & 8 & 6,6 & 8,9 & 9 & 7,8 & 11,3 & 16 & 15,9 & 21,6 \\
\hline davon: & & & & & & & & & \\
\hline laufende Innovationsaufwendungen & 3 & 2,6 & 3,3 & 4 & 3,4 & 5,0 & 3 & 3,3 & 4,2 \\
\hline $\begin{array}{l}\text { Investitionen für Innovationen } \\
\text { darunter: }\end{array}$ & 5 & 4,1 & 5,6 & 5 & 4,4 & 6,3 & 13 & 12,6 & 17,6 \\
\hline FuE-Aufwendungen & 3 & 2,3 & 3,3 & 2 & 1,7 & 2,5 & 2 & 2,0 & 2,7 \\
\hline Investitionen (in Mrd. DM) & 13 & 10,6 & $10,0^{*}$ & 16 & 14,5 & $16,3^{*}$ & 16 & 15,4 & $16,2^{*}$ \\
\hline $\begin{array}{l}\text { Beschäftigte (in Tsd.) } \\
\text { darunter: }\end{array}$ & 672 & 100 & & 722 & 100 & & 894 & 100 & \\
\hline Innovatoren & 496 & 73,8 & 100 & 510 & 70,7 & 100 & 655 & 73,3 & 100 \\
\hline
\end{tabular}




\begin{tabular}{||l|lll|lll|lll||}
\hline \hline darunter: & & & & & & & & & \\
Produktinnovatoren & 466 & 69,4 & 94,0 & 476 & 66,0 & 93,3 & 599 & 67,0 & 91,5 \\
Prozeßinnovatoren & 443 & 65,9 & 89,3 & 435 & 60,3 & 85,3 & 522 & 58,3 & 79,7 \\
FuE-Treibende & 430 & 64,0 & 86,7 & 369 & 51,1 & 72,4 & 501 & 56,0 & 76,5 \\
\hline
\end{tabular}

1) Unternehmen mit mindestens fünf Beschäftigten.

2) 1994 einschließlich Gründungen aus den Jahren 1992 bis 1994.

3) Der Anteil des Umsatzes mit nicht oder nur unerheblich verbesserten Produkten der Innovatoren am Umsatz der Innovatoren bzw. der Anteil der Investitionen der Innovatoren am Umsatz der Innovatoren ist jeweils mit * gekennzeichnet.

Quelle: $\quad$ ZEW (1996): Mannheimer Innovationspanel; entnommen aus Licht, Schnell und Stahl (1996).

\section{Tabelle 4: Kenngrößen zum Innovationsverhalten im westdeutschen Verarbeitenden Gewerbe}

\begin{tabular}{|c|c|c|c|c|c|c|c|c|c|}
\hline & \multicolumn{3}{|c|}{$1994^{2)}$} & \multicolumn{3}{|c|}{1993} & \multicolumn{3}{|c|}{1992} \\
\hline & absolut & in & & absolut & in & & absolut & in & $\%$ \\
\hline Unternehmen $^{1)}$ & 58400 & 100 & & 55800 & 100 & & 59500 & 100 & \\
\hline darunter: & & & & & & & & & \\
\hline $\begin{array}{l}\text { Innovatoren } \\
\text { darunter: }\end{array}$ & 31100 & 53 & 100 & 30500 & 54 & 100 & 36700 & 62 & 100 \\
\hline Produktinnovatoren & 27900 & 48 & 90 & 27100 & 49 & 89 & 32700 & 55 & 89 \\
\hline Prozeßinnovatoren & 25200 & 43 & 81 & 25800 & 46 & 85 & 26000 & 44 & 71 \\
\hline FuE-Treibende & 22900 & 39 & 74 & 15800 & 28 & 52 & 21400 & 36 & 58 \\
\hline FuE-Abteilung & 8500 & 15 & 27 & 8500 & 15 & 28 & 10700 & 18 & 29 \\
\hline $\begin{array}{l}\text { Umsatz (in Mrd. DM) } \\
\text { darunter: }\end{array}$ & 1880 & 100 & & 1856 & 100 & & 1997 & 100 & \\
\hline $\begin{array}{l}\text { Innovatoren } \\
\text { darunter: }\end{array}$ & 1537 & 81,8 & 100 & 1478 & 79,5 & 100 & 1693 & 84,8 & 100 \\
\hline Produktinnovatoren & 1481 & 78,8 & 96,4 & 1415 & 76,1 & 95,7 & 1645 & 82,4 & 97,2 \\
\hline Prozeßinnovatoren & 1400 & 74,5 & 91,1 & 1394 & 75,1 & 94,3 & 1567 & 78,5 & 92,6 \\
\hline FuE-Treibende & 1335 & 71,0 & 86,9 & 1199 & 64,5 & 81,1 & 1415 & 70,9 & 83,6 \\
\hline nach Art der Produkte: & & & & & & & & & \\
\hline neue oder wesentlich verbesserte & 409 & 21,8 & 26,6 & 355 & 19,1 & 24,0 & 331 & 16,6 & 19,6 \\
\hline verbesserte & 361 & 19,2 & 23,5 & 375 & 20,2 & 25,4 & 424 & 21,2 & 25,0 \\
\hline nicht/ nur unerheblich verbesserte ${ }^{3)}$ & 1110 & 59,0 & $\begin{array}{r}49,9 \\
*\end{array}$ & 1126 & 60,7 & $\begin{aligned} 50,6 & \\
* & \end{aligned}$ & 1242 & 62,2 & 55,4 \\
\hline $\begin{array}{l}\text { Innovationsaufwendungen (in Mrd. } \\
\text { DM) }\end{array}$ & 73 & 3,9 & 4,8 & 75 & 4,0 & 5,1 & 93 & 4,7 & 5,5 \\
\hline davon: & & & & & & & & & \\
\hline laufende Innovationsaufwendungen & 52 & 2,8 & 3,4 & 53 & 2,8 & 3,6 & 55 & 2,8 & 3,2 \\
\hline $\begin{array}{l}\text { Investitionen für Innovationen } \\
\text { darunter: }\end{array}$ & 21 & 1,1 & 1,4 & 22 & 1,2 & 1,5 & 38 & 1,9 & 2,3 \\
\hline FuE-Aufwendungen & 55 & 2,9 & 3,6 & 57 & 3,1 & 3,9 & 55 & 2,8 & 3,3 \\
\hline Investitionen (in Mrd. DM) & 74 & 3,9 & $4,0^{*}$ & 85 & 4,5 & $4,7^{*}$ & 97 & 4,9 & $4,9^{*}$ \\
\hline $\begin{array}{l}\text { Beschäftigte (in Tsd.) } \\
\text { darunter: }\end{array}$ & 6472 & 100 & & 6869 & 100 & & 7491 & 100 & \\
\hline
\end{tabular}




\begin{tabular}{||l|rrr|rrr|rrr||}
\hline \hline $\begin{array}{l}\text { Innovatoren } \\
\text { darunter: }\end{array}$ & 5165 & 79,8 & 100 & 5489 & 79,9 & 100 & 6156 & 82,8 & 100 \\
$\begin{array}{l}\text { Produktinnovatoren } \\
\text { Prozeßinnovatoren }\end{array}$ & 4976 & 76,9 & 96,3 & 5303 & 77,2 & 96,6 & 5948 & 79,4 & 96,6 \\
FuE-Treibende & 4657 & 72,0 & 90,2 & 5146 & 74,9 & 93,8 & 5430 & 72,5 & 88,2 \\
\hline
\end{tabular}

1) Unternehmen mit mindestens fünf Beschäftigten.

2) 1994 einschließlich Gründungen aus den Jahren 1992 bis 1994.

3) Der Anteil des Umsatzes mit nicht oder nur unerheblich verbesserten Produkten der Innovatoren am Umsatz der Innovatoren bzw. der Anteil der Investitionen der Innovatoren am Umsatz der Innovatoren ist jeweils mit * gekennzeichnet.

Quelle: $\quad$ ZEW (1996): Mannheimer Innovationspanel; entnommen aus Licht, Schnell und Stahl (1996). 


\section{Literaturangaben}

Barjak, F., J. Felder, A. Fier (1998): Die Treuhand entläßt ihre Kinder der unterschiedliche Werdegang von Westtöchtern und MBO's, Vortragsmanuskript für die Tagung der Gesellschaft für Regionalforschung, Mannheim

Faere, R., S. Grosskopf, M. Norris und Z. Zhang (1994), Productivity Growth, Technical Progress, and Efficiency Change in Industrialized Countries. American Economic Review, Vol. 84, No. 1, S. 66-83.

Felder, J., et al. (1995): Innovationsverhalten der deutschen Wirtschaft: Ein Vergleich zwischen Ost- und Westdeutschland, ZEWDokumentation 95-03. Mannheim

Felder, J. (1998): Analyse wirtschaftlicher Aufholprozesse, Mannheim, mimeo

Gittlemann, M. und E. N. Wolff (1995), R\&D activity and cross-counry growth comparisons. Cambridge Journal of Economics, No.1, S. 189207.

Grossman, G. und Helpman, E. (1993), Innovation and Growth in the Global Economy. Cambridge, Massachusetts and London.

Licht, G., W. Schnell und H. Stahl (1996): Ergebnisse der Innovationserhebung 1995. ZEW-Dokumentation 96-05. Mannheim 